SPIE Smart Structure/NDE 2006, Sensors and Smart Structures Technologies for Civil, Mechanical, and Aerospace Systems, San Diego, CA, 26 February- 2 March, 2006, paper \# SS06-6174 -08

\title{
On the Modeling of Piezoelectric Wafer Active Sensor Impedance Analysis for Structural Health Monitoring
}

\author{
Bin Lin \\ Mechanical Engineering Department, University of South Carolina \\ Columbia, SC 29208, linbin@engr.sc.edu \\ Victor Giurgiutiu, $\mathrm{PhD}$, \\ Mechanical Engineering Department, University of South Carolina \\ Columbia, SC 29208, victorg@sc.edu
}

\begin{abstract}
Structural health monitoring (SHM) is important for reducing maintenance costs while increasing safety and reliability. Piezoelectric wafer active sensors (PWAS) used in SHM applications are able to detect structural damage using Lamb waves. PWAS are small, lightweight, unobtrusive, and inexpensive. PWAS achieve direct transduction between electric and elastic wave energies. PWAS are essential elements in the Lamb-wave SHM with pitch-catch, pulse-echo, phased array system and electromechanical impedance methods.

This paper starts with the state of the art on the impedance method for PWAS applications. Then, finite element impedance model for free and bonded PWAS with different sizes and shapes will be given. Experiments showed that the real part and imaginary part of PWAS had different usage. Applications of impedance-based structural health monitoring indicate impedance method as a good candidate for damage detection and sensor durability verification for SHM smart sensor.
\end{abstract}

Keywords: piezoelectric wafer active sensors, structural health monitoring, impedance

\section{INTRODUCTION}

\subsection{Background}

Impedance-based structural health monitoring techniques have been developed as a promising tool for real-time structural damage assessment, and are considered as a new non-destructive evaluation method. The in-situ impedance is critical for the interfacing of the PWAS with the external electronics. Impedance controls how much energy gets from the electronics into the PWAS and how much energy is extracted back into the electronics.

The electro-acoustical transfer functions of the transmitting and receiving PWAS transducers are determined by the stress and strain coupling between the PWAS and the host structure. Auld (1990) pointed out that it is not sufficient to calculate the amplitude of the radiated acoustic wave in terms of the electric current (or voltage) applied at the input terminals of the transducer, since the acoustic field reacts back on the electrical source and, in this way, affects the level of excitation. This back reaction always occurs when the electrical source has finite impedance. For a PWAS transducer, the input impedance will depend on the intrinsic transducer capacitance $C$ and on the effects of acoustic radiation. Figure la presents a set of experimental results obtained from previous work. The real part of the measured PWAS impedance presents two distinct features: (a) a rather regular pattern of wide peaks and valleys spanning a few hundreds of $\mathrm{kHz}$ each; and (b) dense zigzags of finer peaks riding on them. Intuitively, the wide peaks and valleys are 
associated with Lamb-mode tuning. At the same time, the dense zigzags represent local resonances of the structural modes. However, a comprehensive analytical exploration of this phenomenon does not yet exist. Examination of the specialized literature reveals that a similar phenomenon was observed in connection with Rayleigh-wave interdigitated surface acoustic wave (SAW) devices and delay lines. One difference between the SAW devices and the PWAS transducers is that the former have only one Rayleigh-wave tuning peak, while the latter have several peaks, each associated with a different Lamb mode. Another difference is that the SAW devices do not couple with the underlying structure, while the PWAS transducers couple strongly with the underlying structure and are sensitive to the structural resonances.

(a)

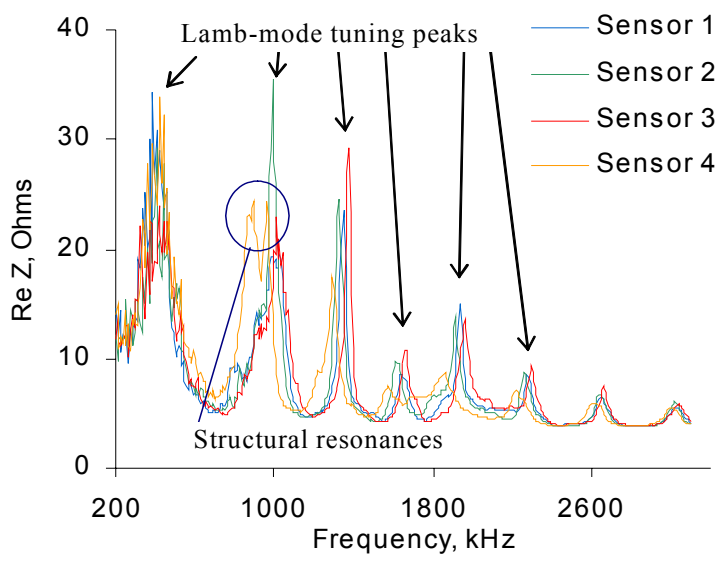

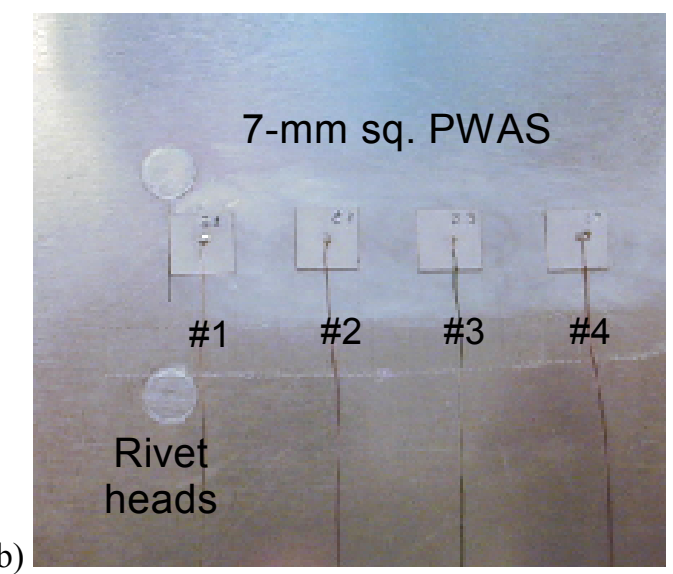

(b) heads

Figure 1 (a) Lamb modes tuning peaks and structural resonances of the PWAS impedance measured in the 200-2600 kHz range on the specimen; (b) The detection of simulated crack damage in aging aircraft panels using the E/M impedance method. (Giurgiutiu et al., 2002)

\subsection{Motivation}

The purpose of this paper is to develop the analysis methods to predict the in-situ impedance of PWAS interacting with structural Lamb waves. Then, these expressions will be used to analyze the power flow and determine the conditions for operation under minimum-power conditions.

\section{STATE OF THE ART}

The theoretical development of the impedance measurements to structural health monitoring was developed by Lian et al. (1994), Sun et al. (1995), Park et al. (1999), Giurgiutiu et al. (2002, 2003).

The impedance of PWAS was analytical modeled for simple structures. Giurgiutiu and Zagrai (2000) gave the detailed derivation of complex formulas for admittance and impedance of piezoelectric bar. They assumed harmonic excitation and considering the 1-D equation of motion, the free PWAS admittance can be described as

$$
Y=\mathrm{i} \omega \cdot C\left[1+\kappa_{31}^{2}\left(\frac{\tan \varphi}{\varphi}-1\right)\right]
$$

where capacitance $C=\varepsilon_{33}^{T} b l / t, \varphi=\frac{1}{2} \gamma l_{a}$, and $\kappa_{13}^{2}=d_{31}^{2} /\left(s_{11} \varepsilon_{33}\right)$ is the electromechanical coupling factor .

Giurgiutiu and Zagrai (2002) determined an analytical expression for PWAS immittance and impedance on standing waves on a 1-D structure undergoing axial and flexural vibrations:

$$
Y=\mathrm{i} \omega \cdot \bar{C}\left\{1-\bar{\kappa}_{31}^{2}\left[1-(\bar{\varphi} \cot \bar{\varphi}+\bar{r})^{-1}\right]\right\},
$$

where $\varphi=\frac{1}{2} \gamma l_{a}, \gamma=\omega / c_{P W A S}$, and $c_{P W A S}$ is the sound speed in the PWAS material. The quantity $\bar{r}=\bar{k}_{s t r}(\omega) / k_{P W A S}$ is the complex stiffness ratio. For standing 1-D waves, they calculated the effective structural stiffness using the 
conventional axial and flexural vibration modes of a 1-D beam, i.e.,

$$
\bar{k}_{s t r}(\omega)=\rho A\left\{\sum_{n_{u}} \frac{\left[U_{n_{u}}\left(x_{a}+l_{a}\right)-U_{n_{u}}\left(x_{a}\right)\right]^{2}}{\omega_{n_{u}}^{2}+2 \mathrm{i} \zeta_{n_{u}}-\omega^{2}}+\left(\frac{h}{2}\right)^{2} \sum_{n_{w}} \frac{\left[W_{n_{w}}^{\prime}\left(x_{a}+l_{a}\right)-W_{n_{w}}^{\prime}\left(x_{a}\right)\right]^{2}}{\omega_{n_{w}}^{2}+2 \mathrm{i} \zeta_{n_{w}}-\omega^{2}}\right\}^{-1}
$$

where $\omega_{n}$ and $\zeta_{n}$ are the modal frequencies and damping ratios, while $u$ and $w$ signify axial and flexural displacements, respectively.

Giurgiutiu and Zagrai considered PWAS radial mode vibration of a thin disk. The 2-D analysis of PWAS immittance for circular-crested Lamb waves were performed in cylindrical coordinates using the Bessel functions formulation. In previous work, the PWAS immittance for circular vibrations, we determined analytical expressions for the admittance and impedance of a PWAS mounted on a 2-D structure undergoing axisymmetric radial and flexural vibrations:

$$
Y(\omega)=\mathrm{i} \omega C\left(1-k_{p}^{2}\right) \cdot\left[1+\frac{k_{p}^{2}}{1-k_{p}^{2}} \frac{(1+v) J_{1}\left(\varphi_{a}\right)}{\varphi_{a} J_{0}(\varphi)-(1-v) J_{1}\left(\varphi_{a}\right)-\frac{a}{r_{a}} \chi(\omega)(1+v) J_{1}\left(\varphi_{a}\right)}\right]
$$

where the stiffness ratio $\chi(\omega)=k_{s t r}(\omega) / k_{P W A S}$ was calculated using the axisymmetric radial and flexural vibration modes, $R_{k}(r)=A_{k} J_{1}\left(\lambda_{k} r\right), \quad Y_{m}(r)=A_{m} \cdot\left[J_{0}\left(\lambda_{m} r\right)+C_{m} \cdot I_{0}\left(\lambda_{m} r\right)\right]$, i.e.,

$$
k_{s t r}(\omega)=a^{2} \rho \cdot\left[\frac{2}{h} \cdot \sum_{k} \frac{\left[r_{a} R_{k}\left(r_{a}\right)-\int_{0}^{a} R_{k}(r) H\left(r_{a}-r\right) d r\right] R_{k}\left(r_{a}\right)}{\left(\omega_{k}^{2}-2 \mathrm{i} \varsigma_{k}+\omega^{2}\right)}+\frac{h}{2} \cdot \sum_{m} \frac{\left[3 Y_{m}\left(r_{a}\right)+r_{a} \cdot Y_{m}^{\prime}\left(r_{a}\right)\right] \cdot Y_{m}^{\prime}\left(r_{a}\right)}{\left(\omega_{m}^{2}-2 \mathrm{i} \varsigma_{m}+\omega^{2}\right)}\right]^{-1}
$$

This equation can be used to predict the admittance and impedance frequency response. As the excitation frequency varies, and resonance and anti-resonance frequencies are encountered. In practice, admittance and impedance magnitudes always display some limited values due to the effects of internal losses inside piezoelectric material.

\section{FINITE ELEMENT ANALYSIS OF PWAS IMPEDANCE}

The analytical model of PWAS impedance has been explored and verified under 1-D linear and 2-D radial mode structure. For more complex structure, the analytical model is hard to be solved. The Finite Element Analysis is a commonly used tool for design and optimization of piezoelectric transducers. It allows the accurate calculation of resonance modes and frequencies as well as impedance spectra. However, the limitation of memory and computing time makes it only useful for small structure. The commercial FEM package ANSYS was used for the modeling described in this work. The 3-dimensional models were built by SOLID5 elements. These are 3-D coupled-field brick elements with eight nodes and six degrees of freedom (3 displacements, voltage, scalar magnetic potential and temperature). Here, only the electromechanical coupling was employed. For the modeling we applied the material parameters of APC-850 (Table 1) provided by APC International Ltd. The nodes of the bottom and top planes were defined as electrodes by a common electrical potential equal to the driving voltage and the ground potential, respectively. The impedance spectra of PWAS were modeled with the harmonic response analysis of ANSYS.

Table 1 Properties of a typical PZT active-sensor wafer (APC-850)

\begin{tabular}{|l|c|c|}
\hline Property & Symbol & Value \\
\hline Compliance & $s_{11}^{E}$ & $15.300 \cdot 10^{-12} \mathrm{~Pa}^{-1}$ \\
\hline Dielectric constant & $\varepsilon_{33}^{T}$ & $15.470 \cdot 10^{9} \mathrm{~F} / \mathrm{m}$ \\
\hline Induced strain coefficient & $d_{13}$ & $-175 \cdot 10^{-12} \mathrm{~m} / \mathrm{V}$ \\
\hline Coupling factor & $\kappa_{31}$ & 0.360 \\
\hline Length-wise wave speed & $c$ & $2900 \mathrm{~m} / \mathrm{s}$ \\
\hline Permittivity of free space & $\varepsilon_{0}$ & $8.84194 \mathrm{pF} / \mathrm{m}$ \\
\hline
\end{tabular}




\subsection{Impedance of Free PWAS}

The analytical model equation (4) can be used to predict the 1-D admittance and impedance frequency response. As the excitation frequency varies, and resonance and anti-resonance frequencies are encountered, the admittance and impedance go through $+\infty$ to $-\infty$ transitions. In practice, admittance and impedance magnitudes always display some limited values due to the effects of internal losses inside piezoelectric material.

Comparison of theoretical, FEM and experimental impedance of free circular PWAS are shown in Figure 2. The analytical and FEM methods can predict the resonance frequency very well. For square PWAS, FEM method impedance spectra agreed with experimental result well.

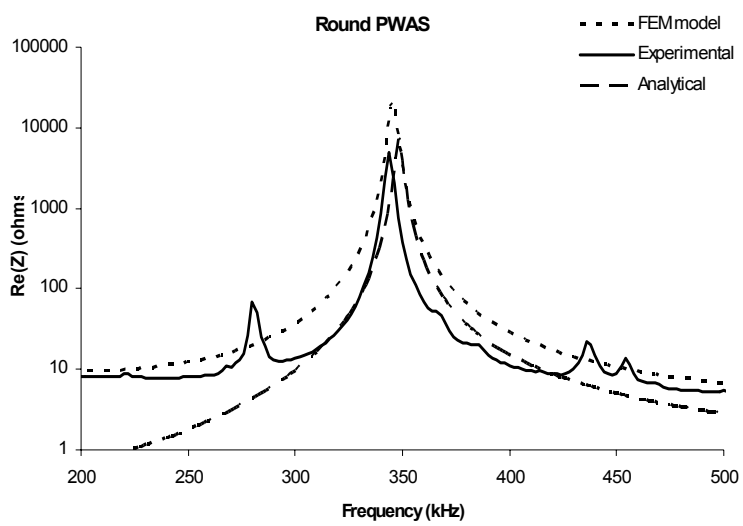

(a)

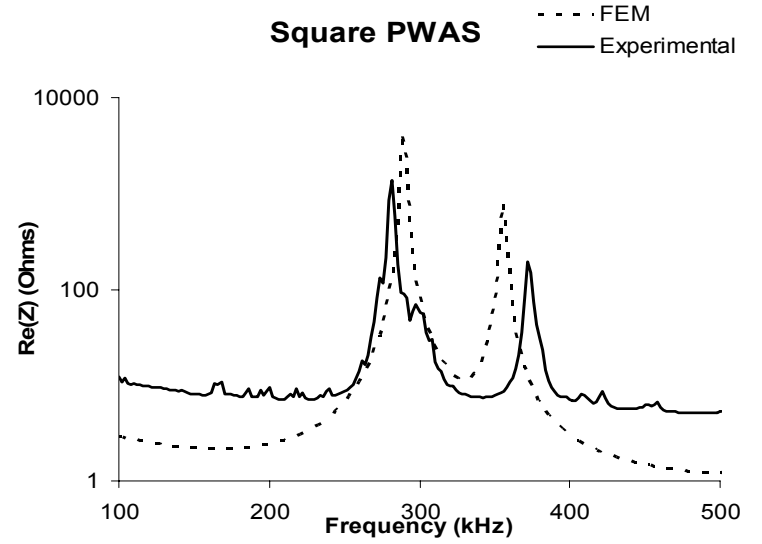

(b)

(a) $7 \mathrm{~mm}$ diameter, $0.2 \mathrm{~mm}$ thickness round APC 850 PWAS; (b) $7 \mathrm{~mm}$ square, $0.2 \mathrm{~mm}$ thickness square PWAS.

\subsection{Impedance of rectangle PWAS}

For rectangular PWAS, both length and width will contribute to impedance resonance frequency. The impedance of length and width can be superposed together to approximately predict the impedance spectrum. Using this concept, the theoretical value for a $25 \mathrm{~mm} \times 5 \mathrm{~mm} \times 0.15 \mathrm{~mm}$ rectangular PWAS was shown in Figure 3. The approximately analytical impedance after superposition of the length and width impedance, the FEM impedance and the experimental impedance spectra are shown in Figure 3. The superposition analytical can predict the impedance of rectangle PWAS at low frequency range. The FEM analysis gives better results.
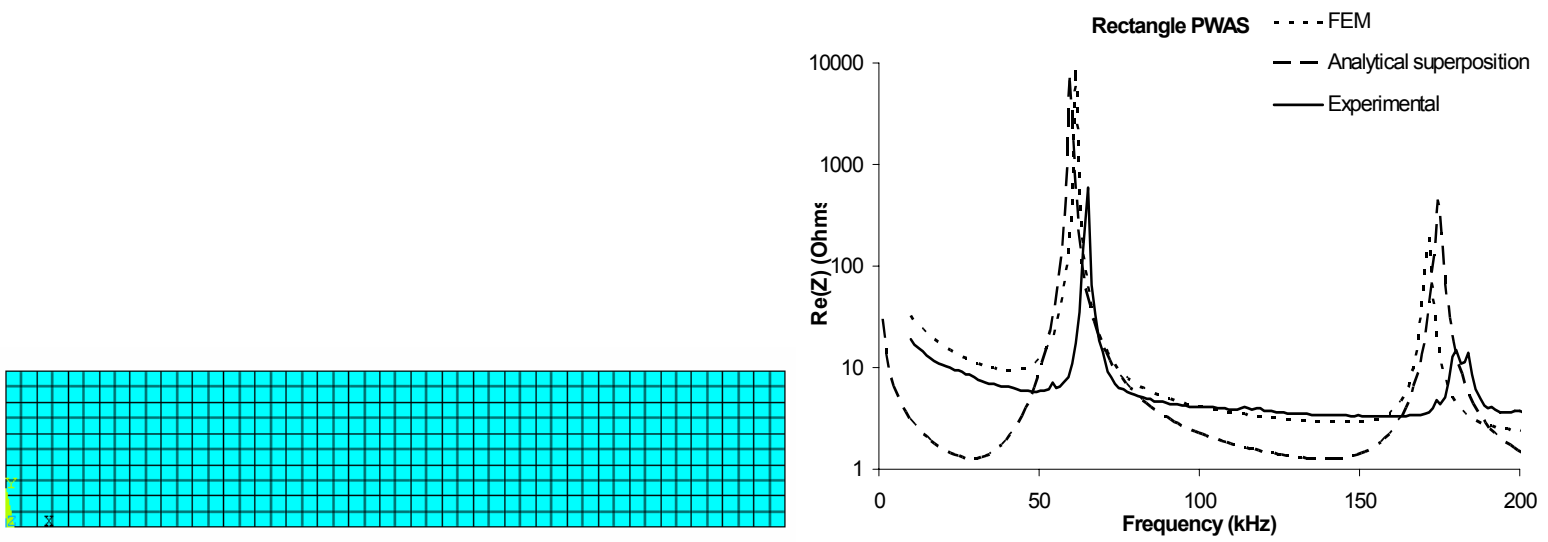

Figure 3 (a) FEM model of a $25 \mathrm{~mm}$ x $5 \mathrm{~mm}$ x $0.15 \mathrm{~mm}$ rectangular PWAS, (b) The approximately theoretical impedance, FEM analytical impedance and the experimental impedance spectra 


\subsection{Impedance of PWAS bonded on a circular plate}

Giurgiutiu and Zagrai considered a thin, isotropic circular plate with a PWAS surface-mounted at its center. Under PWAS excitation, both axial and flexural vibrations are set in motion. The structural dynamics affects the PWAS response modifies its electromechanical impedance. They use 2-D analysis of PWAS and structure impedance to model the interaction between the PWAS and the structure and to predict the impedance spectrum that would be measured at the PWAS terminals during the structural process. The experimental specimen is shown in Figure 4. The analytical calculation predicted the resonance impedance very well. (Figure 5 (a))

The FEM model gave more control ability to predict the impedance spectra. Figure 5 (b) showed FEM model with and without considering the adhesive layer. The adhesive layer reduce the amplitude of impedance and change a little of resonance frequency. The model with the adhesive layer is more accurate and close to the experimental data.

(a)

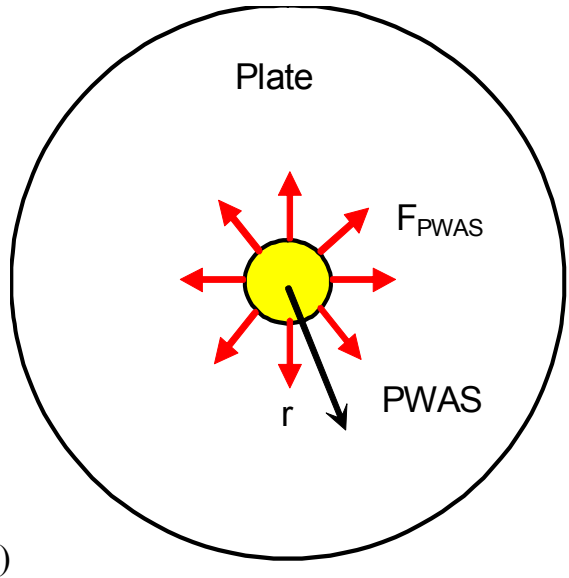

(b)

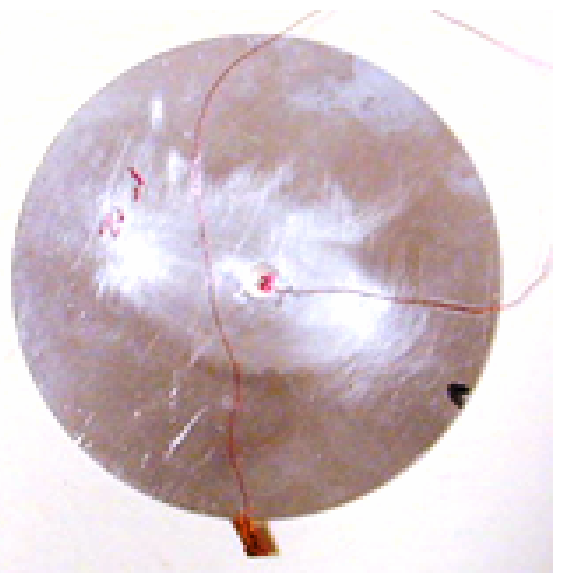

Figure 4 Experimental setup (a) Illustration of PWAS bonded on a circular plate; (b) test specimen.

(a)

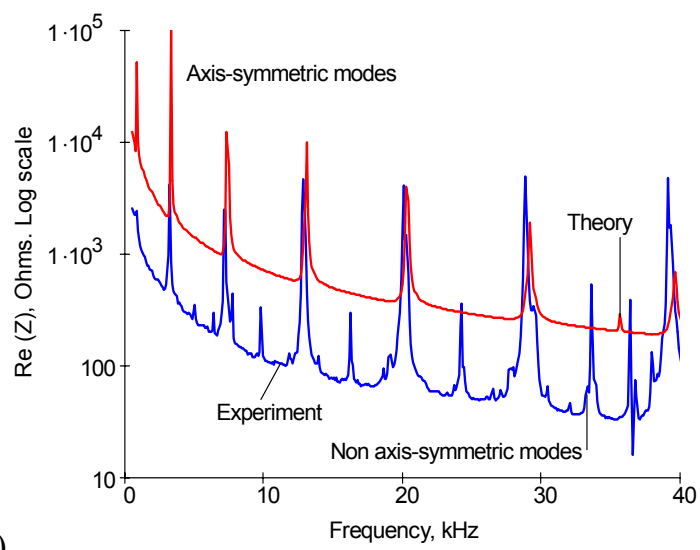

Figure 5 (a) Comparison of experimental and analytical impedance of PWAS bonded on a circular plate (Giurgiutiu et al., 2002); (b) Comparison of experimental and FEM impedance of the same structure.

PWAS can detect subtle changes in the high-frequency structural dynamics at local scales. The local changes in the high-frequency structural dynamics are associated with the presence of incipient damage. Giurgiutiu and Zagrai tested impedance of the simulated crack on circular plates (Figure 6). The analytical model is not applicable for structure with crack. The FEM model can be use to model the incipient damage in this situation. The FEM model impedance with crack is compared with the undamaged FEM impedance. 
(a)

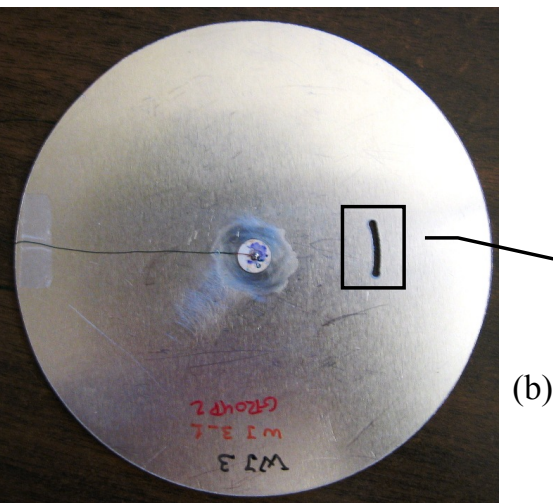

(b)

(c)

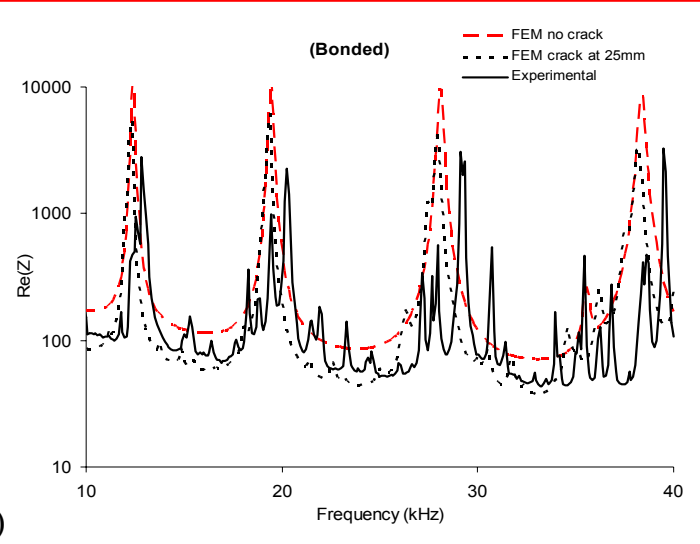

Figure 6 PWAS sample bonded on a circular plate with crack at $25 \mathrm{~mm}$ (b) detail mesh in crack area, (b)

Comparison of the experimental results with FEM model impedance with and without incipient crack.

\subsection{Impedance model of PWAS array}

In damage detection experiment on thin-plate structure by using guided waves, Giurgiutiu and Bao (2001), integrate the embedded ultrasonic sensor radar algorithm into it. For convenience, this system is called EUSR. They used square PWAS to bond on the structure. The sensor layout is shown in Figure 7. The consistence of PWAS bond is essential to the propriety damage detection. We used the impedance method to check the consistence.

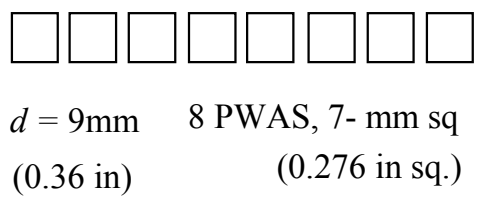

(a)
(0.36 in)
(0.276 in sq.)

Figure 7 PWAS array (a) 9-element PWAS array with 7-mm square PWAS

PWAS array installed one by one (Figure 7) has its advantage; the nearby PWAS in the array will not affect each other. But its disadvantage is that you may not install consistent. An alternative way is to install PZT sheet first and then deposit the electrodes to make them as PWAS arrays. Figure 8 showed the 2-D PWAS array with thirty-two 7-mm square electrodes on top. The 2-D PWAS array has its advantage in installation and consistence but it is more complicated. The nearby PWAS will constrained each other and affect the impedance. To study the constrain affect to the impedance, the FEM models of a single PWAS (no constrain), PWAS electrode at a corner (2-side constrain) and PWAS electrode at center (4-side constrain) were made to analyze the impedance spectra. It is shown in Figure 9(a). The impedance spectra of these models are in Figure 9(b).

(a)

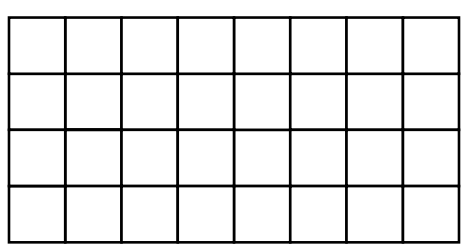

$7 \mathrm{~mm}$ square each (b)

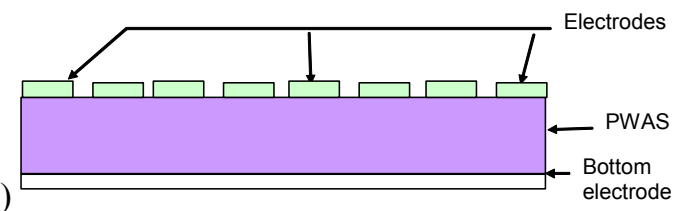

Figure 8 Illustration of 2-D PWAS array (Total size 56 x $28 \mathrm{~mm}$ ) (a) top view; (b) side view. 
(a)
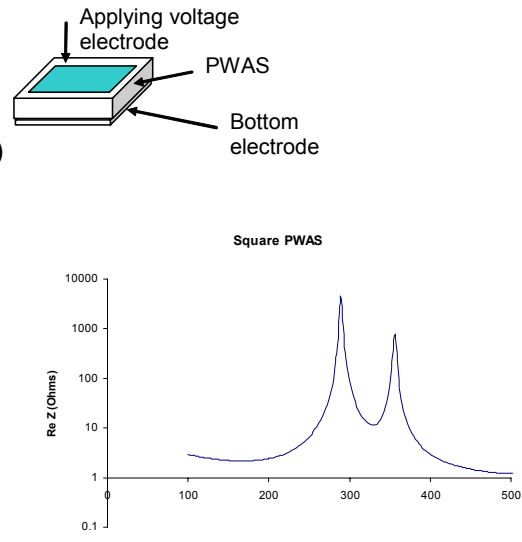

(b)

Figure 9 Model and impedance spectra of the free PWAS array without bonding on plate (a) model of free PWAS , PWAS Electrode at a corner and PWAS Electrode in the center of array (b) impedance spectrum

\section{IMPEDANCE AS QUALITY CONTROL}

\subsection{Impedance Spectrum Check}

The intrinsic E/M impedance and admittance spectra of the PWAS, before being attached to the structure, were measured with the HP 4194A Impedance Phase-Gain Analyzer. Center clamping conditions were simulated and the wafer could perform free vibrations while being tested. To obtain the intrinsic E/M impedance and admittance spectra, the PWAS were tested in the $100 \mathrm{~Hz}-12 \mathrm{MHz}$ frequency range using the HP 4194A Impedance Analyzer. The data was collected through the GPIB interface and processed in MS Excel.

(a)

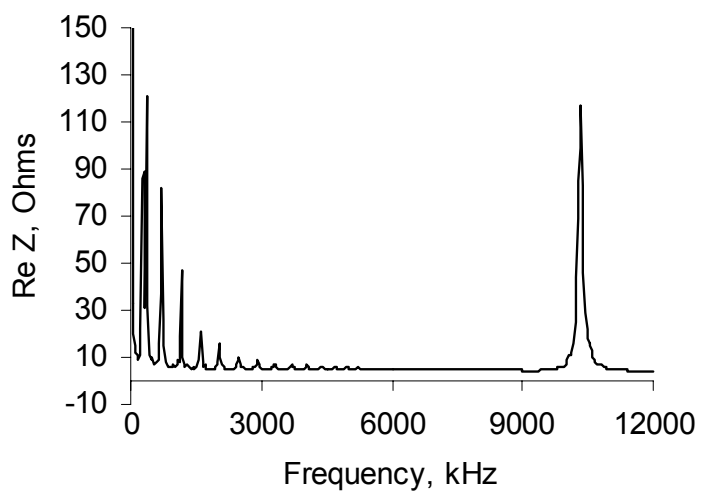

(b)

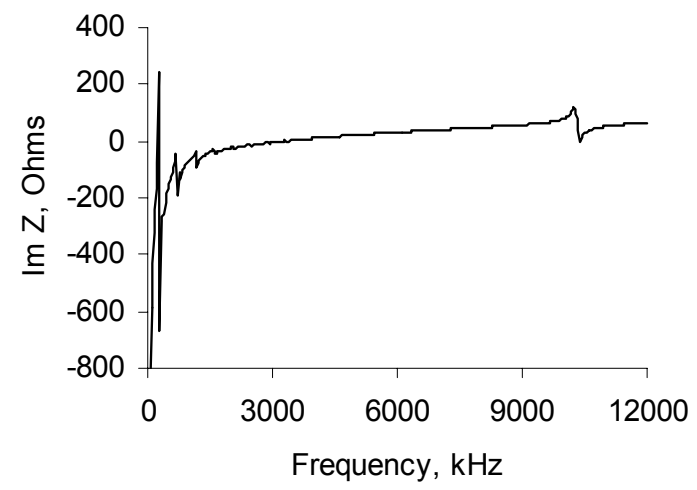

Figure 10 Impedance characteristic of a free-free free $7 \mathrm{~mm}$ square PWAS: (a) real part of impedance; (b) imaginary part of impedance. 


\subsection{Impedance Spectrum Check}

The E/M impedance and admittance spectra of the PWAS, after being attached to the structure, were measured with the HP 4194A Impedance Phase-Gain Analyzer as baseline data. The wafer could perform in-plate vibrations while being tested. To obtain the E/M impedance and admittance spectra, the PWAS were tested in the $100 \mathrm{~Hz}-2 \mathrm{MHz}$ frequency range using the HP 4194A Impedance Analyzer. The data was collected through the GPIB interface and processed in MS Excel.
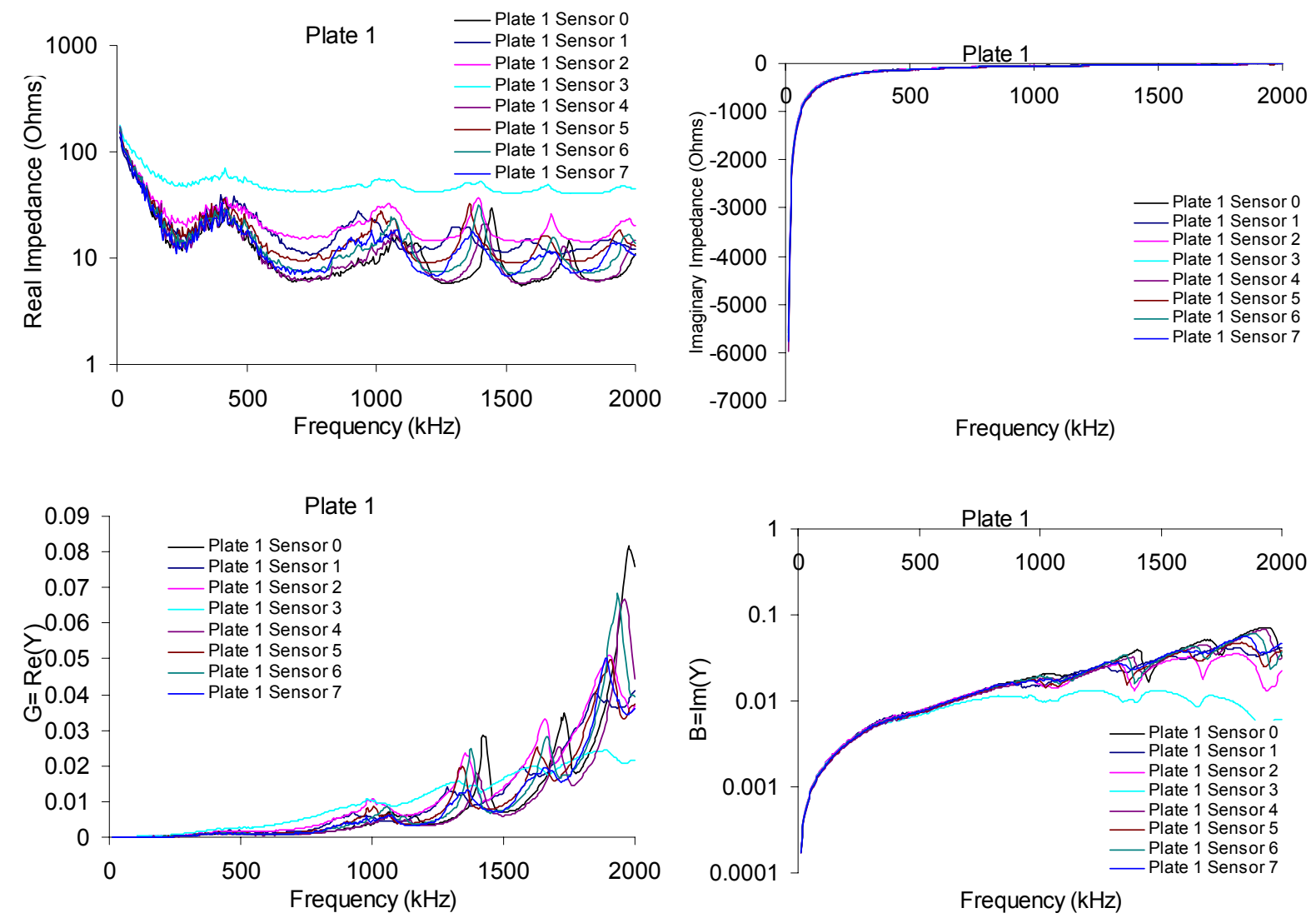

Figure 11 Imaginary Admittance of $7 \mathrm{~mm}$ Square PWAS on Plate 1

Table 2 Max and Min values for the Magnitude of the Impedance and Admittance of the $7 \mathrm{~mm}$ Square PWAS

\begin{tabular}{|c|c|c|c|c|}
\hline \multicolumn{5}{|c|}{ Plate \#1 - 7 mm Square PWAS } \\
\hline \multirow{2}{*}{ PWAS \# } & $|\mathrm{Z}|$ (Ohms) & \multicolumn{2}{|c|}{$|\mathrm{Y}|$ (Siemens) } \\
\cline { 2 - 5 } & Max & Min & Max & Min \\
\hline 0 & 5583.929 & 9.977869 & 0.100254 & 0 \\
\hline 1 & 5543.722 & 16.87878 & 0.058462 & 0 \\
\hline 2 & 5480.842 & 17.92376 & 0.055789 & 0 \\
\hline 3 & 5587.572 & 42.89732 & 0.025299 & 0 \\
\hline 4 & 5972.865 & 11.25974 & 0.088833 & 0 \\
\hline 5 & 5742.533 & 16.1871 & 0.061759 & 0 \\
\hline 6 & 5697.08 & 12.39198 & 0.08075 & 0 \\
\hline 7 & 5759.389 & 14.28274 & 0.070016 & 0 \\
\hline
\end{tabular}




\section{CONCLUSION AND FURTHER WORK}

The finite element method was used to model the free and bonded PWAS. The results agreed very well with the analytical and experimental impedance spectra. A simulated crack was added to the structure finite element models to monitor the impedance spectra change. The next step will consider the effective structural stiffness and impedance presented by the structure to the PWAS when the structure is experiencing propagating waves. This will be achieved by separating the PWAS from the structure, and replacing its effect with interaction forces. For each Lamb wave mode, a corresponding surface velocity will be calculated. The total surface velocity will be determined by the summation of all Lamb modes. Dividing the force by velocity, the effective structural impedance and the electromechanical impedance of the PWAS installed on a structure experiencing propagating Lamb waves will be determined. This approach will be extended by considering the multimodal Lamb waves instead of single-modal axial and flexural waves. We will consider a multitude of Lamb-waves that will be selectively tuned to explain the experimental data.

\section{ACKNOWLEDGMENTS}

This material is based upon work supported by the National Science Foundation under Grant \# CMS-0408578 and CMS-0528873, Dr. Shih Chi Liu Program Director and by the Air Force Office of Scientific Research under Grant \# FA9550-04-0085, Capt. Clark Allred, PhD Program Manager. Any opinions, findings, and conclusions or recommendations expressed in this material are those of the authors and do not necessarily reflect the views of the National Science Foundation or the Air Force Office of Scientific Research.

\section{REFERENCES}

1. Chang, F.-K. (1998) "Manufacturing and Design of Built-in Diagnostics for Composite Structures", 52 $2^{\text {nd }}$ Meeting of the Society for Machinery Failure Prevention Technology, Virginia Beach, VA, March 30 - April 3, 1998.

2. Park, G., et al. (2003) "Overview of Piezoelectric Impedance-Based Health Monitoring and Path Forward", Shock and Vibration Digest, Vol. 35, No. 6, November, 2003, pp. 451-463

3. Wang, C. S.; Chang, F.-K. (2000) "Built-In Diagnostics for Impact Damage Identification of Composite Structures", in Structural Health Monitoring 2000, Fu-Kuo Chang (Ed.), Technomic, 2000, pp. 612-621

4. Giurgiutiu, V and Zagrai, A.N. (2000) "Characterization of Piezoelectric Wafer Active Sensors", Journal of Intelligent Material Systems and Structures, Vol. 11, No. 12, December 2000, pp. 959-976

5. Giurgiutiu, V.; Zagrai, A. N. (2002) "Embedded Self-Sensing Piezoelectric Active Sensors for Online Structural Identification", ASME Journal of Vibration and Acoustics, Vol. 124, No. 1, January 2002, pp. 116-125

6. Lin, X.; Yuan, F. G. (2001a) "Diagnostic Lamb Waves in an Integrated Piezoelectric Sensor/Actuator Plate: Analytical and Experimental Studies”, Smart Materials and Structures, Vol. 10, 2001, pp. 907-913

7. Giurgiutiu, V.; Zagrai, A. N.; Bao, J. (2002) "Embedded Active Sensors For In-Situ Structural Health Monitoring of Thin-Wall Structures”, ASME Journal of Pressure Vessel Technology, Vol. 124, No. 3, August 2002, pp. 293302 